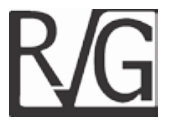

\title{
Resultados monetarios: Escenarios y metodologías de cálculo en economías inflacionarias*
}

\author{
Uzcátegui, Sigilfredo** \\ De La Hoz, Betty***
}

\begin{abstract}
Resumen
Este artículo tiene como objetivo analizar los escenarios de resultados monetarios y las metodologías utilizadas para su determinación. Se trata de una investigación de tipo descriptiva documental sustentada en la Norma Internacional de Contabilidad 29 (IASCF, 2005), así como en los fundamentos teóricos de Catacora (2009), Chillida (2005), Fernández (2002), RomeroMuci (2005), Vives (2003), entre otros. Con base en los casos planteados por Catacora (2009), se analizan los escenarios de resultado monetario del ejercicio, confrontando la teoría con la realidad a la que podría enfrentarse una empresa en épocas inflacionarias, adicionalmente se comparan las metodologías para el cálculo del resultado monetario a través de un caso hipotético. Los resultados evidencian la existencia de todo un panorama de escenarios de resultado monetario del ejercicio; y aunque se utilicen procedimientos diferentes para su cálculo, se obtiene el mismo resultado. Se concluye que el desglose del resultado monetario es un elemento fundamental a considerar en el análisis de la información financiera, a fin de evitar interpretaciones erróneas que pudieran distorsionar la toma de decisiones.
\end{abstract}

Palabras clave: resultado monetario del ejercicio; posición monetaria; ganancia monetaria; pérdida monetaria; inflación

Recibido: 28-11-14 Aceptado: 02-07-15

* Este artículo es resultado del proyecto de investigación "Movimientos y resultados por posición monetaria en empresas del sector construcción" adscrito al Instituto de Investigaciones de la Facultad de Ciencias Económicas y Sociales (FCES) de la Universidad del Zulia (LUZ) y registrado ante el Consejo de Desarrollo Científico, Humanístico y Tecnológico (CONDES).

** Contador Público. Magíster en Gerencia de Empresas, mención: Gerencia Financiera. Docente de La Universidad del Zulia (LUZ) e investigador del Instituto de Investigaciones de la Facultad de Ciencias Económicas y Sociales de la Universidad del Zulia. E-mail: suzcategui@gmail.com

*** Contador Público. Magíster Scientiarum en Gerencia de Empresas, mención: Gerencia Financiera. Docente de la Universidad del Zulia (LUZ), investigadora adscrita al Departamento de Contabilidad y al Instituto de Investigaciones de la Facultad de Ciencias Económicas Sociales de la Universidad del Zulia. E-mail: betty.hoz@hotmail.com 


\title{
Monetary results: Scenarios and methodologies of calculation in inflationary economies
}

\begin{abstract}
The aim of this research is to analyze the monetary results scenarios and the methodologies utilized for its determination. It is a descriptive and documental research sustained in the International Contability Norm 29 (IASCF, 2005), as well as in the theoretical fundamentals of Catacora (2009), Chillida (2005), Fernández (2002), Romero-Muci (2005), Vives (2003), among others. Based on the cases illustrated by Catacora(2009), the scenarios of monetary results from the practice are analyzed, confronting the theory against the reality to which a company could face in inflationary times. Additionally, the methodologies for the monetary results calculation are compared through a hypothetic case. The results demonstrate the existence of a whole panorama of monetary results scenarios from the practice; and though different methods are used for its calculation, the obtained result is the same. It is concluded that the monetary results breakdown is a fundamental element to consider in the financial information analysis in order to avoid wrong misinterpretations that could distort the decision making.
\end{abstract}

Key Words: monetary results from the practice; monetary position; monetary profit; monetary loss; inflation.

\section{Introducción}

El objetivo principal de los estados financieros es suministrar información útil acerca de la situación financiera, la gestión económica y los flujos de tesorería, facilitando la toma de decisiones empresariales, en función tanto de las características cualitativas fundamentales de la información financiera (relevancia y representación fiel), como de las características cualitativas de mejora (comparabilidad, verificabilidad, oportunidad y comprensibilidad).

Ahora bien, en presencia de la inflación, la toma de decisiones resulta compleja dada la distorsión en las cifras presentadas en los estados financieros. Para evitar errores en la toma de decisiones, todas las operaciones empresariales, en un período dado de tiempo, deben ser sometidas a un proceso de ajuste por inflación, con el propósito de que las cifras se expresen en una moneda del mismo poder adquisitivo. El proceso de ajuste por inflación permite determinar la posición monetaria (diferencia entre activos y pasivos monetarios) y, a partir de esta, el Resultado Monetario del Ejercicio (REME). Este último está compuesto por dos grandes resultados: los provenientes de la reexpresión de partidas cuya naturaleza son las propias del negocio; y los originados del tratamiento financiero de los valores nominales, donde la incidencia de la inflación es lo fundamental. Independientemente del método de reexpresión aplicado, dicho resultado debe ser reconocido en los estados financieros al cierre del ejercicio.

Por otra parte, el patrimonio contable resume las variaciones 
efectivas de dichos resultados monetarios, originados por el tratamiento que la gerencia de la empresa le confiere a los valores nominales constantes los cuales, por estar constituidos en derechos y obligaciones dinerarios, no están protegidos contra los efectos de la inflación y, por tanto, sufrirán una variación.

Con base en lo ya planteado, esta investigación se centra en el análisis de los diferentes escenarios de resultados monetarios y las metodologías utilizadas para su determinación. La investigación es de tipo descriptiva documental sustentada en la Norma Internacional de Contabilidad 29 (NIC 29) emitida por el Comité de Normas Internacionales de Contabilidad (IASCF, por las siglas en inglés de International Accounting Standards Committee Foundation) en el año 2005, para luego contrastar los escenarios de REME propuestos por Catacora (2009), con la realidad empresarial. Adicionalmente, a través de un caso hipotético se comparan las metodologías para el cálculo del resultado monetario del ejercicio.

\section{Algunas consideraciones sobre resultado monetario}

La NIC 29 (IASCF, 2005) señala que en un periodo inflacionario toda entidad que mantenga un exceso de activos monetarios sobre pasivos monetarios, perderá poder adquisitivo, $y$ toda entidad que mantenga un exceso de pasivos sobre activos que no están protegidos contra los efectos inflacionarios, ganará poder adquisitivo, siempre que tales partidas no se encuentren sujetas a un índice de precios.
Lo anterior se refleja en la llamada posición monetaria, la cual indica la forma en que la estructura financiera de una empresa se verá afectada por la inflación. Esta se obtiene de la diferencia entre los activos y pasivos monetarios de una fecha determinada (Rodríguez, 1999; Redondo, 2002). Catacora (2009) y Rodríguez (1999) describen dos tipos de posición monetaria: la activa, cuando los activos monetarios son superiores a los pasivos monetarios; y la pasiva, cuando los activos monetarios son superados por los pasivos monetarios.

Por su parte, Redondo (2002) incluye otro tipo de posición monetaria: posición monetaria neutra, presente cuando los activos y pasivos monetarios tienen el mismo valor, es decir, el efecto que genera la inflación sobre las partidas del activo es igual al efecto producido en las del pasivo. También se conoce como posición equilibrada, al ser iguales los activos y pasivos monetarios, la diferencia entre ellos será cero (0), por lo que no se reflejará ganancia ni pérdida monetaria, sino un perfecto equilibrio.

Así, aquellas entidades que tengan una posición monetaria activa generan pérdidas por efectos de la inflación, pero si dicha posición es pasiva originan ganancias (Catacora, 2009; Romero-Muci, 2005). Para Vives (2003), bajo condiciones de inflación, la empresa gana en su pasivo y pierde en su activo, ambos monetarios; dando a lugar a una ganancia o pérdida monetaria por tenencia de activos y pasivos expresados en valor nominal, la cual será una ganancia cuando la empresa sea un deudor neto, es decir, cuando su pasivo sea superior a su activo.

De este modo, el resultado que origina una posición monetaria 
activa o pasiva, de acuerdo a RomeroMuci (2005), constituye la síntesis de las variaciones patrimoniales reales originadas por el mantenimiento, durante un período dado, de partidas que en época de inflación sufren una variación de valor en términos reales.

Romero-Muci (2005), agrega que partidas monetarias originan REME en función automática de la tasa de inflación. Los resultados obtenidos deben ser reconocidos en los estados financieros, indistintamente del método de valoración aplicado. El problema práctico se presenta en el cálculo de las pérdidas o ganancias monetarias, pues normalmente durante un período contable la entidad dispone simultáneamente de activos y pasivos monetarios diversos y su monto cambia permanentemente durante el mismo.

En consecuencia, empresas con REME-Pérdida disminuirían la utilidad reexpresada, en otras palabras, la resultante de la actualización de partidas cuya naturaleza es propia del negocio; pero empresas con REME-Ganancia aumentarían la utilidad reexpresada o reducirían una pérdida actualizada proveniente de las actividades propias del negocio (Chillida, 2005).

Lo anterior confirma que cuando la posición monetaria es activa o pasiva, se produce un resultado monetario. La activa representará una pérdida monetaria para la entidad en épocas de inflación y la pasiva una ganancia. El resultado, bien sea positivo o negativo, se produce de los cambios en el poder adquisitivo de las partidas monetarias activas y pasivas debido a que su valor nominal es constante.

Según la NIC 29 (IASCF, 2005), la pérdida o ganancia por posición monetaria neta puede ser determinada como la suma algebraica, esto es considerando el signo de las cantidades, de todos los ajustes efectuados para la reexpresión de las partidas correspondientes a los activos no monetarios, patrimonio neto, estado de resultados y las correcciones de los activos y obligaciones indexados. Esta pérdida o ganancia puede estimarse también aplicando el cambio en el índice general de precios a la media ponderada, para el periodo, de la diferencia entre activos y pasivos monetarios.

Según Chillida (2005) y Catacora (2009), la obtención de ganancia o pérdida resultante de los activos y pasivos monetarios propiedad de la empresa se calcula por la diferencia entre la posición monetaria neta estimada y la de cierre. Sin embargo, Catacora (2009) es más específico al mencionar los elementos que en el cálculo del resultado monetario intervienen:

- Posición Monetaria Neta Inicial (PMNI): neto de los activos y pasivos monetarios al inicio del período de cálculo del resultado monetario, expresado en unidades monetarias (u.m.) de la fecha del estado de situación financiera.

- Aumentos a la posición monetaria: operaciones que aumentan las partidas monetarias, entre las cuales se tienen ingresos operacionales, excepto realizaciones de ingresos diferidos; aumentos de capital social en efectivo; registro de ingresos diferidos; precio de venta de activos fijos; ingresos por diferencias cambiarias; otros ingresos

- Disminuciones a la posición monetaria: transacciones que disminuyen la posición monetaria, entre las que se encuentran compras de inventarios; gastos de operación; 
adquisición de activos fijos; compra de Inversiones no monetarias; desembolsos por prepagados no monetarios; otros desembolsos

- Posición Monetaria Neta Estimada (PMNE): suma algebraica de los tres conceptos antes señalados; esto es, posición monetaria neta de la contabilidad ajustada por inflación o a valores constantes e indica lo que debería de reflejar en inflación una empresa, a la fecha del estado de situación financiera.

- Posición Monetaria Neta al Cierre o final (PMNC): se determina en la contabilidad histórica y no se ajusta, puesto que sus valores nominales ya están expresados en unidades monetarias a la fecha de cálculo del resultado monetario.

Redondo (2002) es menos explícito pues solo menciona dos elementos necesarios para el cálculo del resultado monetario:

- Posición monetaria neta estimada: representada por la posición monetaria neta del periodo anterior re expresada de acuerdo al índice de inflación del periodo actual; más o menos el movimiento reexpresado de las partidas monetarias del periodo actual.

- Posición monetaria neta actual o al cierre: representada por la posición neta positiva o negativa hallada en base a valores históricos al cierre del periodo actual.

Por su parte, Fernández (2002), explica que para obtener el resultado monetario del ejercicio se resta algebraicamente la posición monetaria histórica y la posición monetaria estimada.

Como puede verse, Chillida (2005), Catacora (2009) y Fernández (2002), difieren con lo establecido en la NIC 29 (IASCF, 2005): el cálculo del resultado monetario debe realizarse con los ajustes en las cifras de las partidas no monetarias antes y después de la reexpresión. Una vez sumadas algebraicamente cada una de las diferencias obtenidas por la reexpresión de los activos y pasivos no monetarios incluido el patrimonio neto y el estado de resultados, se obtiene el REME.

Chillida (2005), Catacora (2009) y Fernández (2002) sólo hacen referencia a una diferencia entre la posición monetaria neta estimada y la de cierre; Catacora (2009) muestra el detalle las partidas que pueden aumentar y disminuir dicha posición, a fin de obtener la posición neta estimada de un periodo. Sin embargo, es importante acotar que el planteamiento de Fernández (2002) cambia por completo el producto de la operación matemática pues no es lo mismo una diferencia en valores absolutos (sin tomar en cuenta los signos) que restar cifras algebraicamente, considerando si son positivas o negativas.

A efectos de presentación, la NIC 29 (IASCF, 2005) establece que la pérdida o ganancia por posición monetaria neta deberá ser incluida en la utilidad neta del periodo y el ajuste efectuado en los activos y obligaciones indexados, se compensará con la ganancia o pérdida en la posición monetaria neta.

Adicionalmente, la NIC 29 (IASCF, 2005) indica que tanto los ingresos y gastos financieros como las diferencias en cambio derivadas por transacciones en moneda extranjera, relacionadas con los fondos prestados o tomados en préstamo, también estarán asociadas con la posición monetaria neta. Con respecto a esto, recomienda a efectos de su presentación en la cuenta de resultados, que tales partidas sean 
Resultados monetarios: Escenarios y metodologías...

Uzcátegui, Sigilfredo y De La Hoz, Betty

agrupadas con las pérdidas o ganancias producto del proceso de reexpresión.

Al respecto, Chillida (2005) considera que el resultado monetario obtenido al final del ejercicio económico, debe reflejarse en el grupo "costo o beneficio integral de financiamiento" del estado del resultado, el cual también incluye los intereses no capitalizables y las diferencias por variaciones en la tasa de cambio de partidas en moneda extranjera.

Los elementos que intervienen en el cálculo del resultado monetario se presentan en el estado de movimiento de la posición monetaria (Catacora, 2009) o estado del resultado monetario del ejercicio (Yanes, 2001); en el cual se parte de la posición monetaria neta inicial, se suman los aumentos y se restan las disminuciones, para obtener la posición monetaria neta estimada, a la cual se le resta la de cierre para determinar el REME.

\section{Escenarios de resultado monetario}

De acuerdo con la metodología establecida por las Normas Internacionales de Información Financiera para la reexpresión global de la información financiera (IASCF, 2005), el resultado monetario se obtiene de las diferencias entre las cifras de las partidas no monetarias antes y después de la reexpresión, las cuales conforman las ganancias $o$ pérdidas por posición monetaria neta. De este modo, sumando cada una de las diferencias obtenidas por la reexpresión de los activos y pasivos protegidos contra la inflación, incluido el patrimonio neto y el estado del resultado, se obtiene el resultado monetario. La NIC 29 (IASCF, 2005) no describe los posibles escenarios del resultado monetario, por el contrario, básicamente los limita a dos: ganancia y pérdida. La ganancia incrementa el patrimonio neto y la pérdida lo disminuye.

Ahora bien, la metodología tradicional para la determinación del resultado monetario ha sido planteada por Chillida (2005), Catacora (2009) y Fernández (2002), quienes consideran que la ganancia o pérdida monetaria resulta de la diferencia entre la Posición Monetaria Neta Estimada (PMNE) y la Posición Monetaria Neta al Cierre (PMNC)

Para efectos de estainvestigación, el análisis de los escenarios de resultado monetario se centrará en lo planteado por Catacora (2009), puesto que si bien muchos autores describen el resultado monetario y sus metodologías de cálculo (Chillida, 2005; Fernández, 2002), no hay indicios de que otros, excepto Catacora (2009), presenten los distintos escenarios posibles de REME.

Según Catacora (2009), una empresa puede hallarse desde el punto de vista del resultado monetario, ante una ganancia o una pérdida monetaria. Dado que este resultado surge de diferencia entre la PMNE y la PMNC, Catacora (2009) identifica ocho posibles escenarios. Sin embargo, su factibilidad en el ámbito empresarial es discutible.

En el primer escenario se parte del supuesto que tanto la PMNE (500 u.m.) y PMNC (800 u.m.) son activas, pero la segunda es mayor a la primera, es decir, a la fecha final del periodo sobre el que se informa, hay más activos monetarios netos que los reflejados por la contabilidad ajustada por inflación; ello supone una ganancia monetaria (300 u.m.). Una PMNC de mayor cuantía que la estimada genera un aumento en el activo neto monetario; 
esto necesariamente se traduce en un incremento de las ganancias o en una disminución de las pérdidas del ejercicio y por ende en un aumento del patrimonio de la empresa.

En el segundo escenario, ambas posiciones monetarias netas activas, sin embargo, a la fecha del período sobre el que se informa, la neta estimada (500 u.m.) excede a la neta final (350 u.m.). La pérdida monetaria generada (150 u.m.) supone una disminución en el activo monetario neto y por consiguiente en el patrimonio de la entidad, al ser insuficiente lo que muestra la contabilidad histórica versus lo que a la fecha del período sobre el que se informa debería de tener.

En el tercer escenario, la PMNE (500 u.m.) es activa y la de cierre es pasiva (400 u.m.), es decir, en lugar de contar con activos monetarios netos, en el histórico se tienen pasivos $\mathrm{Al}$ reconocer la existencia de un mayor pasivo monetario, se está en presencia de una pérdida monetaria, que según Catacora (2009) sería de 900 u.m. Ahora bien, el monto de la pérdida solo debe cuantificarse en 100 u.m. y no en 900 u.m. como lo indica el autor. La metodología tradicional calcula el REME restando los valores absolutos tanto de la posición monetaria neta estimada como de la histórica. En ningún momento hace referencia a la suma de las dos posiciones monetarias. Este cálculo, tal y como se presenta, altera el resultado ya que una pérdida más elevada de lo que debería afectaría considerablemente ganancia o pérdida neta del ejercicio.

En el cuarto escenario, la PMNE (500 u.m.) es activa y la final es cero o equilibrada. Catacora (2009) se basa en la factibilidad de tener una posición monetaria neta equilibrada, es decir, activos y pasivos monetarios iguales.
En efecto, si la contabilidad ajustada por inflación establece que se deberían tener activos netos por 500 u.m. y la contabilidad histórica muestra una posición equilibrada de 0 , se habrá tenido una pérdida monetaria de 500 u.m. Bajo estos parámetros y con la visión de Catacora (2009) focalizada en una factibilidad matemática, reflejaría más bien un caso de escritorio, con muy pocas probabilidades de presentarse en la realidad.

Es preciso señalar, que el concepto de resultado por posición monetaria como se ha definido a lo largo de este trabajo, es un componente característico de las normas establecidas para la elaboración de la información financiera ajustada por efectos de la inflación. El hecho de que las empresas presentan posiciones monetarias activas o pasivas a lo largo de un ejercicio, origina variaciones patrimoniales, por lo que se deduce que sería ficticio pensar en la presencia de una posición monetaria equilibrada durante ese lapso.

En relación con las implicaciones de presentar una PMNC igual a cero (0), teóricamente el efecto que produciría la inflación sobre el activo monetario, es igual al producido sobre el pasivo, por lo tanto, no se genera ganancia ni pérdida. Por otra parte, con respecto a los efectos del ejercicio a la fecha del periodo sobre el que se informa, no habría incidencia alguna por ser monetarias y por consiguiente, no se reexpresan, ya que al final se encuentran expresadas en la unidad de medida corriente a la fecha del estado de situación financiera. Sin embargo, para efectos de la determinación de la PMNE, del periodo siguiente, el monto de la PMNI volverá a ser cero (0).

El quinto escenario supone unas posiciones monetarias netas estimadas 
y al cierre, pasivas, siendo la histórica pasiva (800 u.m.) mayor que la estimada (500 u.m.), lo que origina una pérdida monetaria por efectos de la inflación (300 u.m.). Con respecto al resultado monetario, a pesar de que ambas posiciones monetarias son pasivas, se puede observar que se genera una pérdida de 300 u.m., en vista de que la posición neta final en valor absoluto es más pasiva que la estimada; esto se traduce en un incremento en el pasivo, una pérdida en el resultado monetario, una pérdida en el resultado del ejercicio, y por ende, una disminución en el patrimonio de la empresa.

El sexto escenario, muestra una posición monetaria neta estimada y final pasiva; pero en este caso, la monetaria neta estimada (500 u.m.) es mayor que la de cierre (350 u.m.). En este sentido, existe una disminución neta de pasivos entre la contabilidad ajustada por inflación y la contabilidad a valores nominales o históricos, es decir, se produce una ganancia monetaria (150 u.m.). Se observa claramente una ganancia monetaria ya que se evidencia una disminución neta de pasivos. En otras palabras, la posición monetaria neta final es menos pasiva que la monetaria neta estimada. Frente a este escenario, se podría inferir que los pasivos fueron aplicados en la adquisición o mejoras de activos no monetarios, que no pierden valor con la inflación y serán pagados con unidades monetarias que ya han perdido poder adquisitivo.

En séptimo lugar, se maneja el supuesto que la posición monetaria neta estimada es pasiva y la de cierre activa. Aquí, la contabilidad a valores constantes presenta la posición monetaria neta pasiva (500 u.m.), pero la contabilidad histórica muestra que la de cierre es activa (400 u.m.), por lo que se genera una ganancia monetaria (900 u.m.). A este escenario se da el mismo tratamiento que al número tres, donde efectivamente hay una ganancia, no obstante, solo deben reconocerse 100 u.m. y no 900 u.m., como lo señala Catacora (2009). Para esta situación, la ganancia monetaria debe ser de 100 u.m., ya que el REME se obtiene de la diferencia entre la posición monetaria neta estimada y la de cierre. Considerar 900 u.m. de ganancia alteraría no solo el resultado monetario, sino también, la ganancia o pérdida neta del ejercicio.

El octavo escenario presenta una posición monetaria neta estimada pasiva (500 u.m.) y una de cierre equilibrada, es decir de cero (0). Al igual que en el cuarto escenario, se presenta una situación de equilibrio en la posición monetaria final; sin embargo, si la contabilidad ajustada por inflación afirma que se deberían tener pasivos monetarios pero luego se comprueba que la PMNC es cero, Catacora (2009) considera que se ha originado una ganancia monetaria (500 u.m.). Este escenario es similar al cuarto, con una reducida probabilidad de presentarse en la práctica, por el hecho de ser la posición neta final igual a cero (0). Es decir, el valor del activo y pasivo monetario es igual. En tal sentido, cuando se está en presencia de una economía inflacionaria, necesariamente se piensa en las manifestaciones de posiciones monetarias activas o pasivas durante el ejercicio económico.

A pesar de que Catacora (2009) describe ocho posibles escenarios en los cuales puede encontrarse una empresa con respecto a su resultado monetario, también menciona los casos de una posición monetaria neta estimada equilibrada, y neta al cierre activa o pasiva. Los razonamientos en ambos casos serían similares a los ya 
analizados en los escenarios número cuatro y ocho.

El noveno escenario supone que la PMNE es equilibrada y la de cierre es activa (500 u.m.), de manera que la ganancia monetaria es de 500 u.m. En este caso, la PMNE es igual a cero (0), por lo tanto, es un resultado que difícilmente pueda presentarse en la realidad. Paralelamente, la posición al cierre se presenta activa; esta se determina en la contabilidad histórica no ajustada, y establece que se está en presencia de una ganancia monetaria.

Finalmente, el Catacora (2009) considera un escenario en el que la PMNE sea equilibrada, y la final sea pasiva (500 u.m.). Al igual que en la situación anterior, la PMNE es igual a cero (0), pero en este caso la de cierre es pasiva, por lo tanto, se genera una pérdida monetaria (500 u.m.).

En líneas generales, para el cálculo del REME lo importante es que tanto la posición neta estimada como la de cierre sean activas o pasivas, condición indispensable para que se produzca una ganancia o pérdida monetaria real. Dicho resultado constituye una herramienta fundamental para el análisis de los estados financieros ajustados por inflación y para la toma de decisiones.

Cualquiera sea el resultado, este afecta directamente el patrimonio de la empresa bien sea positiva o negativamente. Lo ideal es tener una ganancia monetaria para que, al momento de trasladar la utilidad o pérdida neta, del estado del resultado al estado de situación financiera, las utilidades acumuladas se incrementen, y por ende, el patrimonio neto.

Las empresas inmersas en economías inflacionarias, pueden afrontar los escenarios de pérdida monetaria, intentando contar con menos activos no protegidos contra la inflación en periodos posteriores, o incrementando sus pasivos al grado de superar los activos monetarios, cuyo valor nominal permanece constante. En estos casos, los gerentes deben ver con mucha cautela el otorgamiento de créditos a los clientes, sobre todo el plazo de cobro establecido, pues la pérdida de poder adquisitivo se incrementa en función de mayor número de días de crédito.

Otra manera de cómo las empresas pueden afrontar escenarios de pérdida monetaria, es obteniendo créditos comerciales y utilizando al máximo su capacidad de endeudamiento. No obstante, la gerencia debe tener especial cuidado con esta estrategia porque si la empresa no puede generar suficiente efectivo para cumplir con la deuda adquirida, y esta se hace cada vez más costosa por las tasas de interés, el endeudamiento en vez de una solución, se convertiría en un problema.

Para facilitar la comprensión de las dos metodologías para el cálculo del resultado monetario, es decir, la global y la tradicional, se presenta un caso práctico, en el que se utiliza información financiera a valores corrientes (históricos) y a valores constantes (reexpresados) de una empresa (Tabla 1). Como información adicional se tiene: al 31-12-20X0 el activo monetario era 2.000 u.m. y el pasivo monetario era 5.150 u.m.; los gastos e ingresos se distribuyen uniformemente durante el ejercicio; y el índice de precios al inicio del período fue 100 , al final del periodo fue 140 y el promedio del periodo fue 120 (Tabla 2). 
Resultados monetarios: Escenarios y metodologías...

Uzcátegui, Sigilfredo y De La Hoz, Betty

\section{Tabla 1 \\ Empresa Ejemplo, C.A. \\ Estado de Situación Financiera \\ Al 31 de diciembre de 20X1 \\ (Expresado en unidades monetarias)}

\begin{tabular}{|c|c|c|c|c|}
\hline & $\begin{array}{l}\text { En moneda } \\
\text { corriente }\end{array}$ & Factor & $\begin{array}{l}\text { En moneda } \\
\text { constante }\end{array}$ & $\begin{array}{l}\text { Variación Posi- } \\
\text { ción Monetaria }\end{array}$ \\
\hline \multicolumn{5}{|l|}{ ACTIVO } \\
\hline Activos no monetarios & 4.000 & $140 / 100$ & 5.600 & 1.600 \\
\hline Activos monetarios & 4.750 & & 4.750 & \\
\hline Total Activo & 8.750 & & 10.350 & \\
\hline \multicolumn{5}{|l|}{ Patrimonio } \\
\hline Capital social & 700 & $140 / 100$ & 980 & 280 \\
\hline Utilidades acumuladas & 300 & $140 / 100$ & 420 & 120 \\
\hline Utilidad neta del ejercicio & 2.000 & & 2.298 & 298 \\
\hline $\begin{array}{l}\text { Posición monetaria neta - } \\
\text { Ganancia }\end{array}$ & & & 902 & 902 \\
\hline Total Patrimonio & 3.000 & & 4.600 & \\
\hline \multicolumn{5}{|l|}{ Pasivo } \\
\hline Pasivo monetario & 5.750 & & 5.750 & \\
\hline Total Pasivo & 5.750 & & 5.750 & \\
\hline Total Patrimonio y Pasivo & 8.750 & & 10.350 & \\
\hline
\end{tabular}

Empresa Ejemplo, C.A.

Estado del Resultado

Por el periodo terminado al 31 de diciembre de 20X1 (Expresado en unidades monetarias)

\begin{tabular}{lrrr}
\hline & $\begin{array}{c}\text { En moneda } \\
\text { corriente }\end{array}$ & Factor & $\begin{array}{r}\text { En moneda } \\
\text { constante }\end{array}$ \\
\hline Ventas & 9.000 & $140 / 120$ & 10.500 \\
Amortización & 150 & $140 / 120$ & 210 \\
Otros gastos & 6.850 & $140 / 120$ & 7.992 \\
Utilidad neta del ejercicio & $\mathbf{2 . 0 0 0}$ & & \\
Posición monetaria neta - Ganancia & & & $\mathbf{2 . 2 9 8}$ \\
Utilidad neta del ejercicio & & & 902 \\
\hline
\end{tabular}

Fuente: Elaboración propia, en base a los autores 


\section{Tabla 2}

Empresa Ejemplo, C.A.

Estado del Movimiento de la Posición Monetaria

Por el periodo terminado al 31 de diciembre de 20X1

(Expresado en unidades monetarias)

\begin{tabular}{|c|c|c|c|c|}
\hline & $\begin{array}{l}\text { En moneda } \\
\text { corriente } \\
\text { al } 31-12-X 0\end{array}$ & Factor & $\begin{array}{l}\text { En moneda cons- } \\
\text { tante al } 31-12-\mathrm{X0}\end{array}$ & $\begin{array}{l}\text { En moneda } \\
\text { corriente } \\
\text { al } 31-12-X 1\end{array}$ \\
\hline Activos monetarios & 2.000 & & 2.800 & 4.750 \\
\hline Pasivo monetario & 5.150 & & 7.210 & 5.750 \\
\hline Posición monetaria neta & -3.150 & 1,4 & -4.410 & -1.000 \\
\hline \multicolumn{5}{|l|}{$\begin{array}{l}\text { Partidas que } \\
\text { incrementan } \\
\text { la posición monetaria }\end{array}$} \\
\hline Ventas & 9.000 & & 10.500 & \\
\hline \multicolumn{5}{|l|}{$\begin{array}{l}\text { Partidas que disminuyen } \\
\text { la posición monetaria }\end{array}$} \\
\hline Gastos & 6.850 & & 7.992 & \\
\hline $\begin{array}{l}\text { Posición monetaria neta } \\
\text { estimada }\end{array}$ & & & -1.902 & \\
\hline $\begin{array}{l}\text { Posición monetaria neta } \\
\text { al cierre }\end{array}$ & & & -1.000 & \\
\hline $\begin{array}{l}\text { Resultado monetario } \\
\text { del ejercicio (Ganancia) }\end{array}$ & & & 902 & \\
\hline
\end{tabular}

Fuente: Elaboración propia

Considerando la metodología descrita en párrafos anteriores, el cálculo de la posición monetaria neta, en su forma tradicional, resulta de la diferencia entre la PMNE y la PMNC. En el ejemplo, se observa que la PMNE pasiva es de 1.902 u.m. y la PMNC pasiva es de 1.000 u.m.; de la diferencia entre dichos valores se obtiene una ganancia monetaria de 902 u.m. Esto se corresponden con el sexto escenario descrito por Catacora (2009); tanto la PMNE como la PMNC son pasivas, siendo la primera mayor que la segunda. Lo anterior supone una disminución neta de pasivos entre la contabilidad ajustada por inflación y la contabilidad a valores nominales o históricos, produciéndose una ganancia en el resultado monetario del ejercicio.

Ahora bien, con la reexpresión global de los estados financieros, se obtienen diferencias en las cifras de las partidas no protegidas contra los efectos inflacionarios, antes y después de la reexpresión. De este modo, sumando cada una de las diferencias obtenidas por la reexpresión de los activos y pasivos no monetarios incluido el patrimonio neto 
y el estado del resultado, se obtiene la ganancia o pérdida por posición monetaria neta. En el ejemplo, pueden observarse variaciones por posición monetaria que resultan de la diferencia entre los valores corrientes y constantes de activos no monetarios (1.600 u.m.), capital social (280 u.m.), utilidades acumuladas (120 u.m.) y utilidad neta del ejercicio (298 u.m.), las cuales generan un REME de 902 u.m., esto es, una ganancia que incrementa la utilidad neta del ejercicio reexpresada.

Adicionalmente, existe otra forma de calcular el REME no contemplada en la metodología tradicional, ni en la norma internacional. Se trata de una metodología derivada de todos los importes que se incluyen en el estado de situación financiera reexpresado, es decir, los activos y pasivos monetarios $y$ no monetarios $y$ el patrimonio; a valores constantes. Se aplica la ecuación fundamental de contabilidad, determinando la diferencia entre el total de activos y el total de pasivo más patrimonio.

En el ejemplo, la diferencia entre activos (10.350 u.m.) y el pasivo más patrimonio (9.448 u.m.) genera un REME de 902 u.m.; de igual manera, de la suma de la utilidad neta del ejercicio a valores constantes (2.298 u.m.) y la ganancia monetaria (902 u.m.) se obtiene la utilidad neta del ejercicio después de la reexpresión (3.200 u.m.).

Así pues, cualquier metodología descritas empleadas para el cálculo del resultado monetario, conduce al mismo valor. Entonces, sea que se utilice la metodología tradicional, la metodología global o la propuesta en esta investigación, el resultado monetario del ejercicio, producto de la reexpresión, siempre será el mismo.

\section{Resultado monetario y toma de decisiones}

Para Nava (2009) la importancia del análisis de la información financiera radica en la identificación de los aspectos de liquidez, solvencia, endeudamiento, eficiencia, rendimiento y rentabilidad, facilitando la toma de decisiones gerenciales, económicas y financieras en la actividad empresarial. No obstante, se debe considerar el comportamiento de variables exógenas a la actividad empresarial como la inflación, la cual afecta el valor actual de mercado de los activos y pasivos; algunos de ellos, generalmente, se omiten o son valuados al costo de adquisición depreciado; por lo tanto, es necesario reexpresar los estados para lograr un análisis más completo y real de la situación de la empresa.

Para una adecuada toma de decisiones en economías inflacionarias, el análisis de los estados financieros debe realizarse en función a cifras actualizadas. En vista de la relevancia que tienen dichos informes para los diferentes usuarios externos, tales como inversionistas, bancos, acreedores y entes gubernamentales, es necesario conocer la situación financiera y económica real, de lo contrario, las decisiones tomadas serían incoherentes y riesgosas.

Puesto que la inflación es un factor que escapa del control de la empresa, los gerentes financieros recurren a estrategias para minimizar su impacto en los resultados financieros. La inflación causa incertidumbre, de allí que una buena gerencia financiera no espera de brazos cruzados adversidades futuras, sino más bien, se enfoca en qué hacer para no verse golpeada, desprotegida e indefensa ante los efectos inflacionarios. 
Según Ramírez et al (2002), debido a la crisis originada por la inflación y otros factores macroeconómicos, las funciones de tesorería en las empresas cobra mayor importancia. Ellas incluyen política de crédito y cobranzas, estimación de ingresos y desembolsos, flujos de efectivo, financiamiento y colocación a corto plazo, manejo de operaciones bancarias, políticas para asumir niveles de riesgo, centralización/descentralización de la toma de decisiones, entre otras.

La gerencia tiene ante si retos importantes, entre los cuales se encuentra la generación de información financiera de calidad, pues representa la base del proceso racional de toma de decisiones, dentro de los parámetros de oportunidad, objetividad y fines empresariales. Tales decisiones abarcan aspectos como por ejemplo evaluación de proyectos, oportunidades de inversión, elaboración de presupuestos, pago de dividendos, determinación de una tasa efectiva de impuestos, entre otros; además, se dificulta la comparación de los estados financieros en diferentes periodos.

Una vez realizado el proceso de reexpresión de los estados financieros, la gerencia tiene a su disposición el resultado monetario del ejercicio, información suficiente para conocer si su patrimonio neto se afectará positiva o negativamente. La misma permite la toma de ciertas decisiones con respecto a su activo y pasivo monetario en periodos posteriores.

Analistas financieros, tales como Lazzati y Ponte (1990) explican que el análisis del resultado monetario implica su discriminación por fuente generadora. Dicho resultado suele comprender la pérdida monetaria ocasionada por el financiamiento a los clientes; la ganancia monetaria por las deudas comerciales y financieras; y las demás pérdidas y ganancias monetarias. Pero dichos activos y pasivos ocasionan resultados financieros nominales (explícitos o implícitos). Por lo tanto, para cada clase de ellos el resultado real está dado por la diferencia entre el efecto monetario y el resultado financiero nominal respectivo.

Tomando esto como referencia, la primera preocupación de la gerencia debe ser si el estado del resultado al final del período contable se aproxima o no a la realidad; seguida de la capacidad de los activos de la empresa y los efectos producidos por la forma cómo éstos han sido financiados, bien sea con capital propio o ajeno. Sobre esta base, se podría llegar a la conclusión que la empresa presenta una situación financiera favorable si no fuera por el alto costo de un excesivo financiamiento. Pero se pudiera estar cayendo en una interpretación errónea.

Por ejemplo, una empresa pudiera tener un buen margen de utilidad bruta, intereses provenientes del costo financiero ya pagados, y una ganancia monetaria. Sin embargo, cuando el análisis se enfoca en el resultado monetario, es posible que el examen de la pérdida monetaria originada por financiar clientes indique que el margen de utilidad bruta no es tan bueno como parecía o la ganancia monetaria por las deudas financieras muestre que el costo neto no es tan alto como se pensaba.

En tal sentido, si dicha empresa genera al final del período contable pérdida neta, la gerencia pudiera llegar a la conclusión que ésta se originó por el costo desmesurado del financiamiento de terceros y que el margen bruto es razonable, pudiendo cubrir adecuadamente los gastos. Pero, un análisis más profundo orientado a la descomposición del resultado monetario permite a una interpretación más acorde 
con la realidad para la toma de decisiones adecuadas.

Si la gerencia decide no conformarse con la cifra que arroja el margen bruto y el resultado neto del ejercicio, y desagrega el resultado monetario, puede encontrarse con que el financiamiento provisto por bancos y otras instituciones financieras es mucho más elevado que el generado por los proveedores. Ahora bien, si no se dispone del desglose de los intereses implícitos, el resultado monetario revelaría una modificación considerable del margen de utilidad bruta y una disminución importante del costo del financiamiento proveniente de los bancos, situación que conduciría a toma de decisiones erradas.

\section{Conclusiones}

El resultado monetario es un concepto exclusivo de estados financieros reexpresados, correspondientes a empresas que presentan información financiera en moneda de una economía inflacionaria. Así pues, constituye el resumen de todas las variaciones patrimo-niales, producto del tratamiento dado a aquellas partidas que en escenarios inflacionarios varían de valor en términos reales, en relación al índice general de precios.

En economías inflacionarias, la reexpresión de estados financieros permite mostrar la realidad patrimonial; la homogenización de todas las partidas; y la determinación de las ganancias o pérdidas monetarias, reconociendo que en un ambiente inflacionario, la gestión económica, está compuesta realmente por el resultado proveniente de las operaciones propias de la empresa, y por el resultado monetario producto de la inflación.

Además, toda operación entre una partida monetaria y una no monetaria origina una variación en la posición monetaria neta y solo cuando la posición monetaria es activa o pasiva, se produce un resultado monetario y por ende una variación en el patrimonio. Por lo tanto, para efectos de los resultados de esta investigación, la posición monetaria equilibrada o nula no aplica, ya que cuando se habla de inflación en una economía, necesariamente se piensa en las manifestaciones de posiciones monetarias activas o pasivas durante el periodo contable.

A fin de facilitar la aplicación de correctivos, se hace necesario el análisis e interpretación de las cifras reexpresadas, evaluando su origen, las partidas que intervienen en el cálculo del resultado monetario, y su comportamiento durante el período. Además, dada la calidad de la información suministrada por el REME y su importancia en la determinación de las ganancias o pérdidas netas del ejercicio, debería ser impostergable su seguimiento permanente.

Por último, para que se dé un proceso de toma de decisiones adecuado, es necesario que la informa-ción financiera se actualice por efectos de la inflación. Al reexpresar las cifras históricas se genera un resultado, el cual permite analizar detalladamente la capacidad de los activos de la empresa y los efectos producidos por la forma cómo estos han sido financiados. Si no se considera en el análisis de los estados financieros el desglose del resultado monetario, se pudiera caer en interpretaciones erróneas que finalmente distorsionarían la toma de decisiones.

\section{Referencias bibliográficas}

Catacora, Fernando (2009), Reexpresión de Estados Financieros, Red contable de publicaciones. Venezuela. 
Chillida, Carmelo (2005), Análisis e interpretación de Balances. Universidad Central de Venezuela, Ediciones de la Biblioteca - EBUC, Segunda edición, Caracas, Venezuela.

Fernández, Jesús (2002), Reexpresión de Estados Financieros en Venezuela, Enfoque Práctico, La Editorial Maracaibo, S.A., Venezuela.

International Accounting Standards Committee Foundation (2005), Norma Internacional de Contabilidad 29 (NIC 29).

Lazzati, Santiago y Ponte, Jorge (1990), Contabilidad Gerencial e Inflación, ediciones Macchi, segunda edición, Buenos Aires

Nava, Marbelis (2009), Análisis financiero: una herramienta clave para una gestión financiera eficiente, Revista Venezolana de Gerencia, Año 14, № 48, Venezuela, Centro de Estudios de la Empresa, Universidad del Zulia, pp $606-628$.

Ramírez, Guillermina; Solórzano, Lissette R. y Rosillo Canales, Yusmelis (2002), Las Pequeñas y Medianas Empresas (PYMES) en Cumaná Venezuela: inflación, gerencia y desarrollo,
Revista Venezolana de Gerencia, Año 7, No 19, Venezuela, Centro de Estudios de la Empresa, Universidad del Zulia, pp 390-402.

Redondo, Ángel (2002), Curso práctico de contabilidad general superior, Tomo II, Tercera edición, Venezuela.

Rodríguez, Luis Antonio (1999), La influencia de la inflación en la contabilidad, Trabajo final nivelatorio de la contabilidad, Disponible en: http:// www.oocities.org/eureka/plaza/6171/ links/inflac.html. Fecha de consulta: 14 de septiembre de 2007.

Romero-Muci, Humberto (2005), La racionalidad del sistema de corrección monetaria fiscal. Editorial Jurídica Venezolana, Venezuela.

Vives, Antonio (2003), Evaluación financiera de empresas. El impacto de la devaluación y la inflación, Editorial Trillas, S.A. México.

Yanes, José Alberto (2001), Guía de los ajustes por inflación financieros y fiscales, Clemente Editores C.A., Venezuela. 